\title{
Ophthalmic pain following cataract surgery: a comparison between local and general anaesthesia
}

\author{
P Koay, A Laing, K Adams, S Branney, J Mathison, F Freeland, M Studley, H Black
}

Royal Infirmary, Edinburgh

P Koay

A Laing

K Adams

S Branney

J Mathison

F Freeland

M Studley

H Black

Correspondence to: P Koay FRCS, FCOphth, Princess Alexandra Eye Pavilion, Chalmers Street, Edinburgh EH3 9HA.

Accepted for publication 13 September 1991

\begin{abstract}
Ophthalmic pain following uncomplicated extracapsular cataract surgery was assessed postoperatively in 61 patients; $55 \%$ undergoing ophthalmic surgery had no pain or discomfort postoperatively, and $32 \%$ reported slight discomfort. Approximately $8 \%$ of patients reported mild pain and the remaining 5\% experienced moderate to severe pain. Local anaesthesia was shown to be more comfortable postoperatively than general anaesthesia in the immediate postoperative period with both groups receiving similar amounts of postoperative analgesics.
\end{abstract}

The actual appreciation of pain is both subjective and personal to the individual patient. Pain relief following ophthalmic surgery is often prescribed by one profession, assessed and administered by another often with the prescriber having little or no notion of what the patients are actually
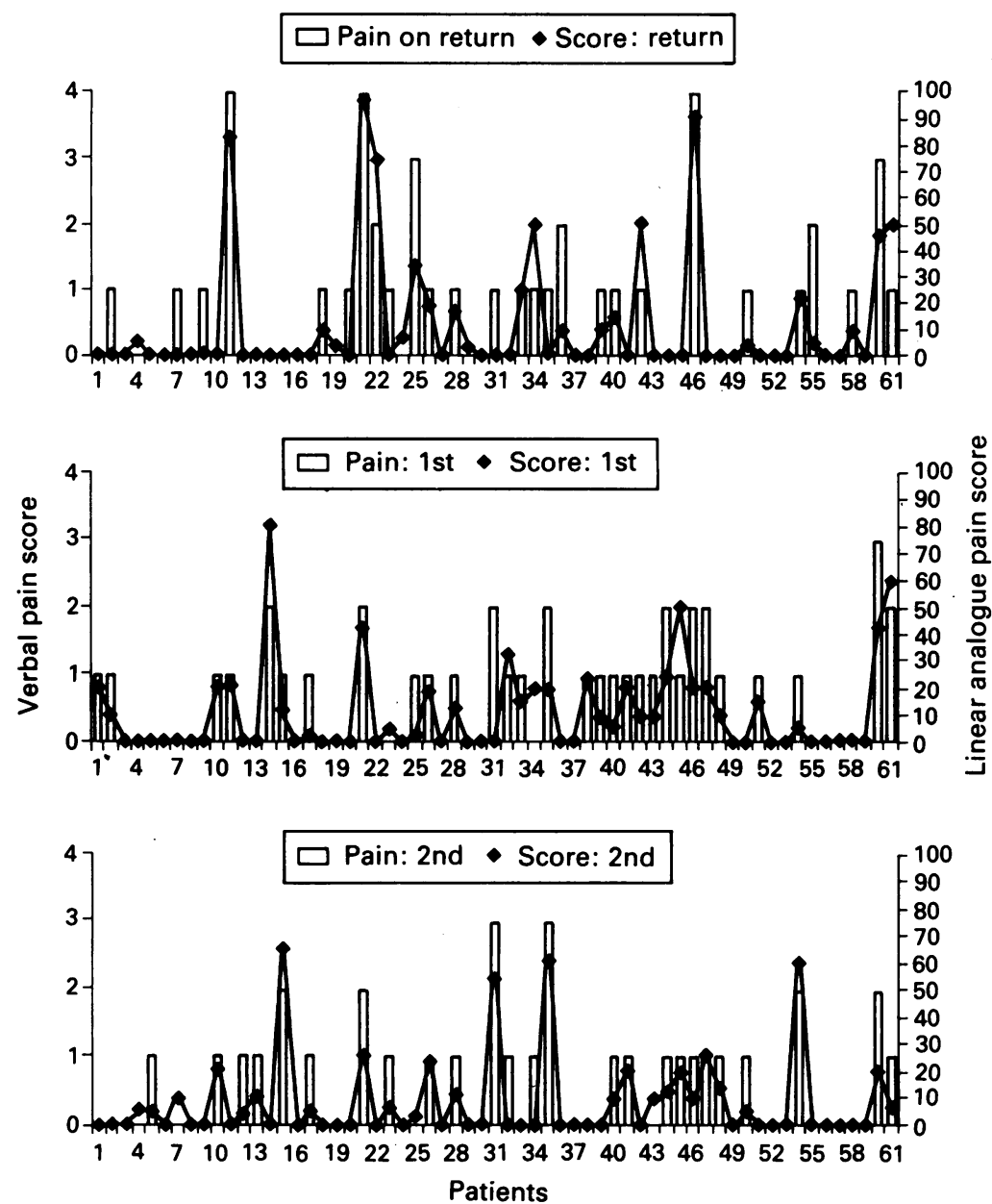

Figure 1 Levels of pain following cataract surgery on day of return to ward (top), first postoperative day (middle), and second postoperative day (bottom).

Table 1 Verbal descriptive pain scoring

$0=$ no pain or discomfort

$1=$ slight discomfort that is tolerable

$2=$ mild pain that would require relief by 'pain-killers'

$3=$ moderate pain that would require relief by 'pain-killers'

$3=$ moderate pain that would require relief by 'pain-killers'
$4=$ severe distressing pain requiring urgent relief by strong 'pain= severe distlers'

Table 2 Linear analogue pain scoring in millimetres

No pain |_ Max pain

Table 3 Nurse or patient's own assessment of anxiety

$0=$ calm, relaxed, not worried

$1=$ anxious, slightly worried about operation

$2=$ very anxious, worried about operation

Table 4 Pain scores

\begin{tabular}{llll}
\hline \multicolumn{4}{c}{ Number of patients } \\
\cline { 2 - 4 } & $\begin{array}{l}\text { Day of return } \\
\text { to ward }\end{array}$ & $\begin{array}{l}\text { lst post-op } \\
\text { day }\end{array}$ & $\begin{array}{l}\text { 2nd post-op } \\
\text { day }\end{array}$ \\
\hline Score 4 & 3 & 0 & 0 \\
Score 3 & 2 & 1 & 2 \\
Score 2 & 3 & 8 & 4 \\
Score 1 & 19 & 21 & 19 \\
Score 0 & 34 & 31 & 36 \\
\hline
\end{tabular}

suffering except the belief that patients are not in pain unless they complain and that perhaps pain is an inevitable consequence of surgery. This study provides an estimate of the levels of postoperative pain encountered in ophthalmic patients which can be compared with other types of surgery measured on a similar standard pain scoring scale.

\section{Method}

Seventy one patients (65\% females, $35 \%$ males) with a mean age of 67 years (SD 16) undergoing extracapsular cataract extractions were consecutively recruited into the study. Assessment of any ocular pain before operation was performed and four patients with pre-existing pain were excluded. The level of pain was quantified by a verbal descriptive scale from 0 to 4 (Table 1) and also on a linear analogue scale of 0 to 100 (Table 2). ${ }^{123}$ Preoperative assessment also included the patients' assessment of their own anxiety level and the nurse assessment of the patients' anxiety level; both using the same verbal anxiety scoring from 0 to 2 (Table 3 ). The patients were also asked to score the level of pain they expected after their operation using the same verbal descriptive pain scoring system. Details of premedications and local anaesthetic type were noted. The extracapsular cataract extractions with intraocular lens (IOL) implants were performed by 16 different surgeons from registrar to consultant grades. 


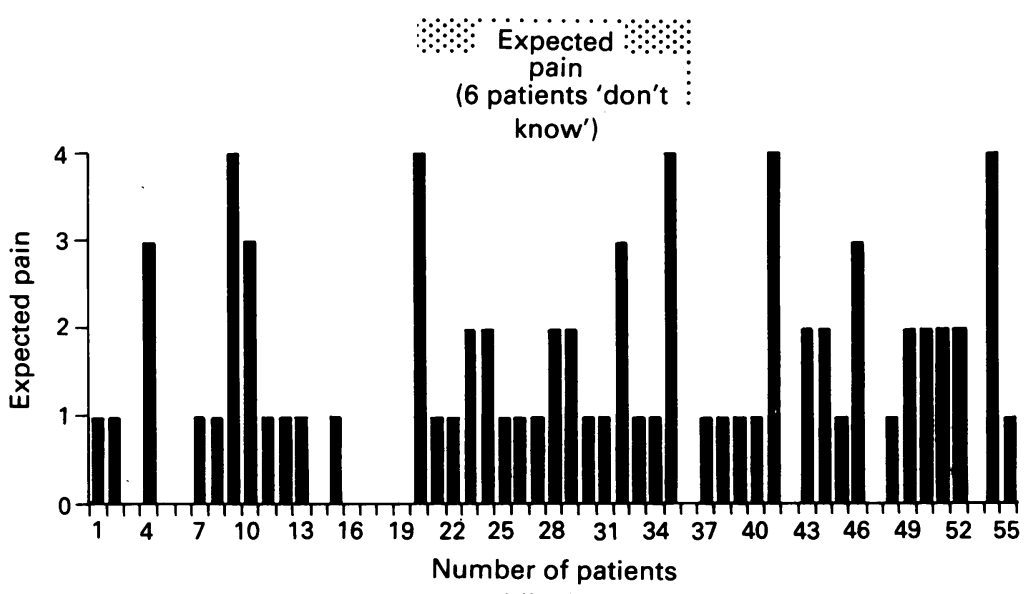

Figure 2 Level of expected pain in patients following cataract surgery.

Pain scores were assessed on return to the ward as soon as the patient was able to cooperate on the day of the operation and in the afternoon of the first and second postoperative day between $2 \mathrm{pm}$ and $4 \mathrm{pm}$ in the afternoon. Nurse assessment of the patient's anxiety level using the same verbal anxiety scoring from 0 to 2 had been obtained on each of the postoperative day. All postoperative complications were recorded and six patients with complications such as corneal abrasions, ocular hypertension requiring intervention and severe iritis were excluded because of possible contribution to postoperative pain. All patients received paracetamol as required with stronger analgesics when necessary. All analgesics dispensed both in theatre by the anaesthetists and in the wards by the nurses for each day were documented.

\section{Results}

The results of the verbal and linear analogue pain scores of 61 uncomplicated extracapsular cataract extractions are shown in Figure 1 and Table 4. There was no significant difference between the pain scores obtained on the day of operation, the first, and second postoperative days. The level of expected pain (Fig 2) was higher than the actual

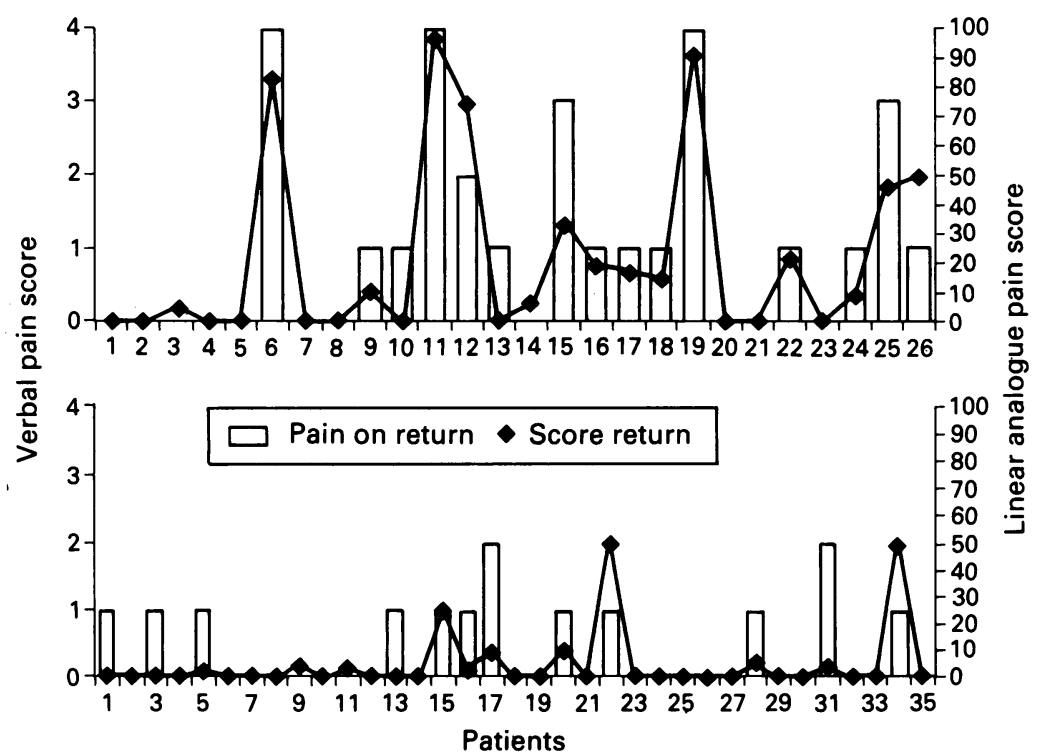

Figure 3 Levels of pain following cataract extraction with general anaesthesia (top) and local anaesthesia (bottom) on day of return to ward. pain scored subsequently in our patients but there is no statistically significant relationship between expected and actual pain scores.

The pain scores of 35 patients under local anaesthesia and 26 patients under general anaesthesia on the day of cataract surgery are illustrated in Figure 3. The level of pain scored was significantly less in the local anaesthesia group compared with the general anaesthesia group both on verbal and on linear analogue scales with $\mathrm{p}=0.018$ and $\mathrm{p}=0.003$ respectively (Wilcoxon signed rank test). Analgesic consumption of paracetamol was also lower in the group having local anaesthetic cataract extractions (mean of $1.4 \mathrm{~g}$ of paracetamol) compared with the general anaesthetic (mean of $2.5 \mathrm{~g}$ of paracetamol) although this was not statistically significant $(p=0 \cdot 20$, Wilcoxon signed rank test).

\section{Discussion}

The results show that in the studied patients about $55 \%$ undergoing ophthalmic surgery have no pain or discomfort postoperatively and approximately $32 \%$ reported slight discomfort. However approximately $8 \%$ of patients reported mild pain and the remaining $5 \%$ experienced moderate to severe pain while on analgesics prescribed when required. There appears to be a small group of patients who may be suffering unnecessarily and may not in fact request analgesics. These patients could benefit from regular dispensing of analgesics.

Patients having local anaesthesia for cataract extraction in this study are more comfortable postoperatively on the day of the operation and require less analgesia. It is of interest that our findings are supported by a German study on postoperative pain. Schaffer and his colleagues in 1988 found that postoperative pain following intraocular surgery was significantly higher after general anaesthesia compared with local anaesthesia with no difference in analgesic therapy in both groups. ${ }^{4}$

One possible explanation for this is that the patients undergoing local anaesthesia are in fact a different population group being older and perhaps less anxious. The mean age of local and general anaesthetic groups of patients were however similar ( 71.8 and 72.6 years respectively). Although the mean anxiety scores of the general anaesthetic patients were higher than those of the local anaesthetic patients $(1.0$ and 0.38 respectively) with $\mathrm{p}=\mathbf{0 . 0 7}$ (Wilcoxon signed rank test) before the operation, those obtained after the operation were similar $(0.5$ and 0.2 respectively) with $p=0.5$ (Wilcoxon signed rank test). This suggests that while general anaesthetic patients were apparently more anxious than local anaesthetic patients before the operation, after their operation there was no difference in their anxiety levels to account for the difference in postoperative pain levels.

As the average analgesic consumption was similar in both the general and local anaesthetic groups $(1.8 \mathrm{~g}$ and $1.7 \mathrm{~g}$ of paracetamol) respectively on the day of return to the ward, with only one general anaesthetic case receiving the opioid analgesic Omnopon (papaveretum) $10 \mathrm{mg}$ intramuscularly, the patients undergoing local anaes- 
thesia for cataract extraction are probably more comfortable postoperatively because of the residual effects of the local anaesthetic agent itself. In all cases, lignocaine $2 \%$ with 1:200000 adrenaline was used as the local anaesthetic agent which may have effects beyond 1 hour, providing patients with useful postoperative analgesia.

The effects of premedication may also contribute to local anaesthesia being more comfortable, with $84 \%$ of the local anaesthetic patients receiving Omnopon $10 \mathrm{mg}$ and the sedating antihistamine Phenergan (promethazine hydrochloride) $50 \mathrm{mg} 1$ hour preoperatively. The premedication for general anaesthesia consists of either diazepam $10 \mathrm{mg}$ or temazepam $10 \mathrm{mg}$ without any analgesia in all cases.

Our findings suggests that there may be a case for administering topical anaesthetic agents to the eye or analgesics for all the general anaesthetic patients before they leave theatre and that regular administration of a mild analgesic, such as paracetamol, in all patients may reduce the overall pain and discomfort in both groups of patients.

We would like to thank Dr B Fleck and Dr B Dhillon for reviewing the manuscript.

1 Huskisson EC. Measurement of pain. Lancet 1974; ii: 1127-31. 2 Scott J, Huskisson EC. Graphic representation of pain. Pain

3 Alexander JI, Hill RG. Pain, the size and measure of the problem. In: Postoperative pain control. Oxford: Blackwell,

4 Schaffer J, Mehrmann M, Heymann-Schramm S, Werry H, Piepenbrock S. Perioperative anxiety and postoperative pain suppression in intraocular operations using general anaesthesia and local anaesthesia. Anaesthesist 1988; 37: 19-23. 